Presented at the 1979 Particle Accelerator

Conference, San Franciscu, CA, March 12-14, 1979

LBL-8961

\title{
THE BEVALAC RADIOTHERAPY FACILITY
}

J. R. Alonso, J. Howard and T. Criswell

March 1979

Prepared for the U. S. Department of Energy under Contract $W-7405-E N G-48$

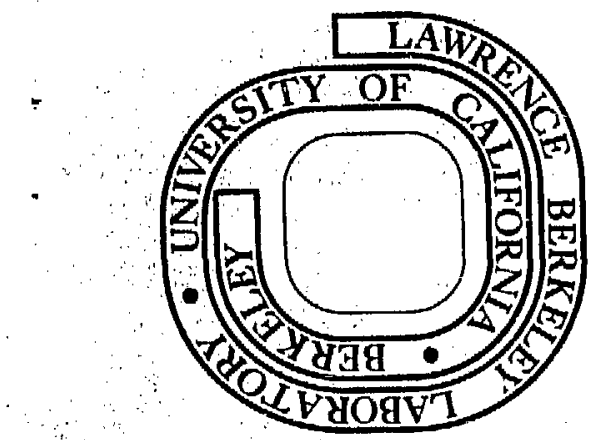




\section{ABSTRACT}

The Patient Treatment Room at the Bevalac is now in full operation. In the design of this ficility, emphasis has been placed on creating an atmssphere appropriate to a clinical facility; the usisal features of an irradiation cave have been hidden behind carpets, curtains and paint. Patient positioning is done with a Philips Ram-style couch, with additional fixtures to accommodate a patient in the sraterd or standing, as well as the supine, position. Dusimetry apparatus, collimators, ion chambers and the beam flattening system used to produce the highly uniform $20 \mathrm{~cm}$ diameter therapy field are described.

\section{INTRODUCTION}

The potentially great advantages to using high LET (Linear Energy Transfer) radiation for the treatment of cancer have been well demonstrated in clinical studies with neutrons, negative pi mesons and heavy ions at several institutions around the world. Studies are underway to explore these possibilities and develop appropriate ter:innlques for optimum utilization of these treatinent midalities.

This paper will discuss the facilities developed at LBL for patient treatment with heavy ion beams from the Bevalac.

Heavy ions offer two distinct advantages over photons: physical dose distribution and enhanced biological ef fectiveness. The siarpness of the characteristic heavyion range-ionization curve, called the Bragg curve (sharp peak in Figure 4) allows substantial sparing of normal tissue, both on the way into the tumor as well as in a very low exit dose beyond the tumor. The rigidity of heavy ion beams also means that tine lateral edges of the irradiation field will be very sharp, at most a few mm wide. The utilization of these physical characteristics will be discussed below, under Beam Delivery. The biological advantages stem from the high ionization density at the end of the particle range (Bragg peak) which causes more permanent cell damage (1ess repair can occur) and so is more lethal to the tissue in which it stops. The lower ionization density along the entrance path is less damaging, and 50 more sparing of nornal tissue.

These properties have been extensively studied at the Bevalac over the last four years with cell and animal radiobiology using beams of $\mathrm{C}$, Ne and Ar. ${ }^{2}$ The understanding gained from these studies has launched a full program of treatment of human cancers.

\section{TREATMENT FACILITY}

The facility layout is shown schematically in Figure 1. In addition to the treatment roum, there is a staging area for physicians, physicists and technicians, an exam room, changing and waiting areas. Since a full patient reception and processing center is located in another nearby buildigg, the patient waiting areas at the Bevalac are relatifely modest.

Full control of all phasts of the treatment, positioning, dosimetry, beam monitorins, and control is done through *Work supported by the Division of Biology and Environmental Research of the Depariment of Energy. the POP 11/45 system in the Biomed control room where a full-time operator is in constant communication with the treatment room and the tecimician's control station.

The treatment room itself is designed to appear like a clinical area. Carpets, curtains and paint hide the usual features of the experimental caves. The curtain through which the beam passes to reach the patient (Figure 2) conceals much of the dosimetry and bean preparation hardware. The final range modulator (water column), ion chambers and collimators are set on the optical rails which are aligned with high accuracy ( $10.2 \mathrm{~mm}$ ) along the leam axis and can translate along this axis a total distance of 2 meters. The drive mechanism is below floor level.

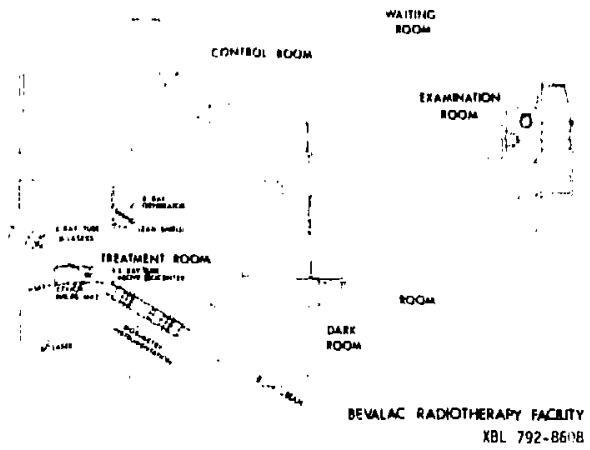

Fig. 1. Schematic layout of the Radios nerapy facility shoving the treatment room, and ancillary areas. The area marked "room" houses the technician's control station, viewing boxes and a discussion area for physicians and physicists.

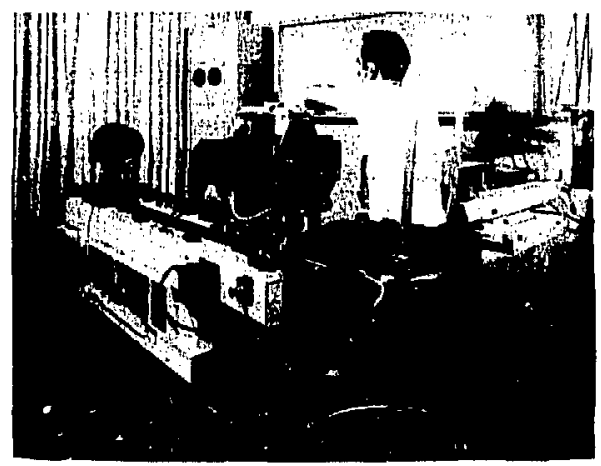

Fig. 2. Treatment room showing chair attachment to the Philips positioner. Beam comes through the curtain and passes through dosimetry apparatus before reaching the patient. Shown on the optical rails are an ion chamber, two collimators and the range-modulating water column. 


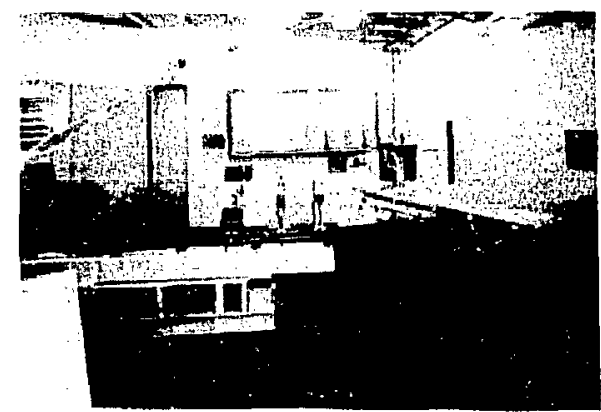

Fig. 3. Treatment room showing table-top assembly supplied with the Philips positioner.

To compensate for the lack of flexibility in beam delivery orientation (we must treat with a static hor (zontal beam) a very versatile patient positioning assembly has been installed, This unit, a Philips Mark I Ram-styla positioner, has four fully motorized motions, whose -oordinates can be read and controlled by computer. It was supplied with a removable table top (Figure 3). At LBL we have fabricated a chair (Figure 2) and a platform for seated and standing treatment modes.

Patient localization with respect to the beam axis is performed using five cross-hair lasers, two installed at lateral locations, one directly on the healn axis and one overhead. All the laser beams are adjusted to pass directly through isocenter. $x$ ray ubes are also located along the beam axis, and at lateral and overhead positions.

\section{BEAM DELIVERY}

The basic tasks in delivering heavy ion radiation to the tumor site are to adjust the beam range so that particles stop at all point.s within the tumor, and to spread the beam out so that all areas of the tumor receive the same dose.

The basic range-energy curve (Bragg curve) is shown as the solid line in Figure 4. Typically, the width of this curve is measured as less than $1 \mathrm{~mm}$. To spread out this peak, the beam is passed through a device known as a Ridge Filter. 4,5 It consists of a brass plate with a precisely machined spiral groove. The exact shape and depth of the groove determine the form of the spread-out Bragg curve. The dotted curve in Figure 4 is a typical example of a modified Bragg curve. The shape of the desired Bragg curve is determined from the results of biology experiments, since one is searching for equal biological effectiveness over the entire range of the spread-out Bragg peak. This "iso-effect" criterion is the reason that the dotted curve in Figure 4 is not flat.

A whole family of ridge filters has been fabricated to cover the full range of treatment situations, namely different degrees of beam spread for each of a number of beam particles and energies.

To facilitate biology and dosimetry studies, certain fixed beam energies were selected. Fine adjustments in the beam range, to assure that it stops in the tumor,
are performed with a computer-controlled water column. 4,5 This instrument consists of two lucite plates driven by precision lead screws. As they move apart, water is drawn into the area between them, forming a carefully controlled variable degrader. The thickness of water can be varied in $0.1 \mathrm{~mm}$ steps from 0 to $30 \mathrm{~cm}$. The water column can be seen on the optical rails in front of the patient in Figure 2 .
The lateral spreading of the beam into a uniform field is done with the scattering-foil occluding-ring system used at Harvard ${ }^{6}$ and at the LBL 184" cyclotion" (see Figure 5). The beam is scattered into a symintric Gaussian distribution by the first scattering ioil (in our case typically $0.6 \mathrm{~cm}$ of lead) located 10 met.ers frori the patient. After travelling 4 meters, thi: Gaussian beam has a half-width of about $4 \mathrm{~cm}$. At ihis point a set of concentric brass rings blocks out cer: tain portions of the distribution. A ridge filter, along with an additional 4 . rm of brass, is attached to these rings to form the second scatterer, acting as a diffuser to give angular spread to the beam. As the beam moves towards the patient, the blocked-out regions are filled in by the scattered beam, and at the patient site the total beam profile is completely flat with an intensity variation of no more than $\pm 2 \%$ over a $20 \mathrm{~cm}$ diameter field. Furthermore, $40 \%$ of the original beanl intensity lies within this flat region, providing excellent utilization of the available beam.

As one might anticipate, the setup and tuning of this system to achieve the quoted flatness is a sensitive procedure. Small misaligninents of the beam on the occluding rings can cause substantial skewing of the intensity profile. 6 To help in the tuning and beamflatness verification a sophisticated proportional chamber called MEOUSA was built. This instrument, described in detail in the following paper, 8 is a

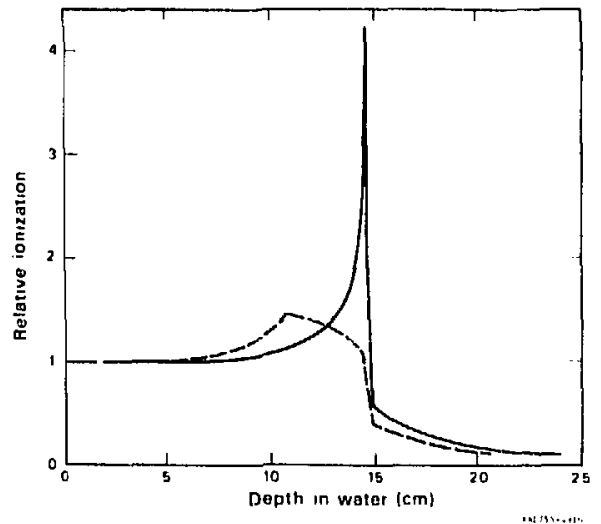

Fig. 4. Range-energy curves measured for a typical heavy ion beam at the Bevalac; the unmodified curve (solid line) shouing characteristically sharp stopping point, and the spread-out curve (dashed line) resulting from passage through the ridge filter. The ionization beyond the stopping point in both curv-s is due to nuclear projectile fragments, which have longer ranges than the primary beam.

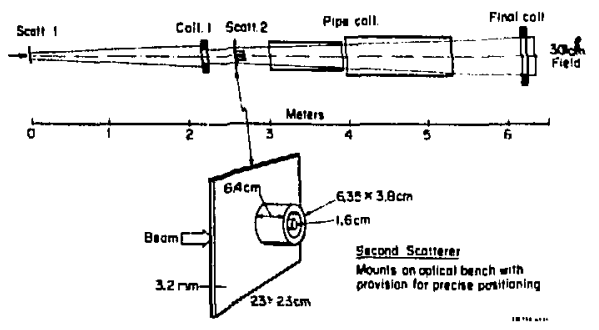

Fig. E. Beam flattening system used at the LBL 184" cyclotron and the Bevalac. Occluding rings block out portions of the Gaussian-shaped beam. Scattering plus drift length combine to produce a very uniform distribution at the site of the patient. 
multi-plane multi-wire chamber which uses CT alyorithms to reconstruct its data into \& $64 \times 64$ picture of the beam profile passing through the chamber. The high precision and fast reconstruction speed make this an ideal device for tuning and verification of the treatment field.

\section{DOSIMETRY AND CONTROL}

Dosimetry is performed with three ion chambers, 4,5 one located upstream of the occluding rings, the other two clcse to the patient. They are calibrated daily for each patient setup with an EG\&G standardized ion chamber. One of the two downstream chambers is read independently of the conputer by a count-down scaler, and serves to terminate the treatment should a computer mal function occur.

The control software (a program called HIRAD) on the POP $11 / 45$ systern monilors and provides archival storage for all of the treatment parameters, including ion chambers, beam intensity, water column setting, ridge filter roiation, patient couch coordinates, etc. It also controls the startup and termination of treatment, removing the beam plug to bring beam into the treatment area. When the desired dose is achieved, a signal is sent which aborts the Bevatron spill (within 1 msec) while the beain plug is inserted back into the line. Elaborate safety provisions have been implemented to provide redundancy and protection against system failures. An operator constantly monitors the computer display during treatment and cun activate emergency shut-offs should he detect any abnormality. This crashoff capability is also available at the radiological technician's station.

\section{RUNNING EXPERIENCE}

The patient treatment program has now been in operation six months. We have treated primarily with carbon beams at $400 \mathrm{MeV} / \mathrm{amu}$, but have also performed treatments with neon and argon beams. Typical Bevalac carton heams are quite intense, up to $\angle$ to $3 \times 10$ To particles ver spill, which translates into a dose rate of uver 400 rads per minutc over the entire $20 \mathrm{~cm}$ diametar treatment field. This rate is sich that a typical treatment is completed in miubir iess than one minute.

One of the reasons for concentrating on carbon ions has been the availability of sceadily improved beam intensities from the local $20 \mathrm{MeV}$ injector. The latest output is $4 \times 10^{9}$ particles per pulse at the patient (almost 100 rads per minute), adequite for most treatments. This redundancy of injectors adds substantially to the beam-delivery reliability.

Machine-time scheduling to accommodate the radiotherapy program has had a substantial impact on the conduct of nuclear science experiments. The machine is reserved for therapy four day-shifts each week, leaving a block of 11 contiguous shifts each weekend for a long nuclear science run. The evening week-day shifts are used for machine studies, radiobiology or nuclear science. Two shifts for maintenance are taken from the nuclear science period on alternate weeks. This schedule permits patients to receive four treatments per week; a full course of treatments will ultimately consist of tiventy iractions.

At present three treatments per day are conducted, but as experience is gained and procedures improved a full patient load of seven to ten per day is anticipated.

\section{FUTURE PLANE}

We anticipate substantial efforts in the future to develop and improve beam delivery systems. The great alignment accuracy required for the occluding-ring flattening system can be avoided by using a beam wobbler. This device consists of a rotating dipole field (provided by a stationary sextupole magnet driven with 3 phase $60 \mathrm{~Hz}$ AC power) which sweeps the beam in a circular pattern at the patient. Field size is provided by appropriate settings of magnet current and the thickness of a scattering foil lacated at the magnet.

Further in the future is a full three dimensional beam scanning systeil. ${ }^{9}$ capable of sweeping a tightly-froused beam across the desired treatment volume. This scanning system will be controlled by a computer which: receives input directly from treatment-planning and CT scanner computers, thus providing the radiotherapist with a completely automated path from diagnostics to treatment. He already have constructed a prototype scanrifing magnet and power supply, and will be testing it shortly.

in summary, we view our radiotherapy facility as a protutype, in which the developments, techniques and experience gained in our day-to-day operation will provide invaluable insights into how to bring heavy-ion therapy most effectively into the hospital environmerit..

\section{ACKNOWLEDGMENTS}

This project would not have been possible without the help of many people. We wish to thank especially the following: Vern Fletcher, Bob Edwards, Ed Stuart and Lou Sylvia for the design and installation of the mechanical components of the treatment area; Jack Henderson, Alan Butler and Dave Redman for the electrical work; John Lyman, Rol in Armer and Frank Upham for instrumentation concepts, design and implementation; Jorge Llacer, Sally Schlaer, R.P. Singh and Paul Barale for computer hardware and software work; and Marsh Tekawa and the Bevatron operators for ever working to improve the beam quality and intensity. Finally we should like to thank Bob walton for the important liaison role he has played between us and the radiotherapy group to help establish design $\mathrm{cri-}$ teria and parameters for this facility.

\section{REFERENCES}

1. 2nd Int. Conf. Particles and Radiation Therapy; Int, J. of Rad. Oncolayy, Biol.-Phys. Vol. 3 , (1977) Pergamon Press, N.Y.

2. Biol. \& Med. Research with Accelerated Heavy Ions at the Bevalac, 1974-77, LBL-5610, April 1977.

3. Philips Medical Systems, 710 Bridgeport Ave., Shelton, Conn. 06484.

4. J.T. Lyman and J. Howard, p. 81 of Ref. 1,

5. J.T. Lyman and J. Howard, p, 26 of Ref. 2.

6. A.M. koehler, R.J. Schneider, J.M. Sistersan, Med. Phys. 4, (1977) p. 297.

7. K. Crowe, L. Kanstein, J.T. Lyman, F, Yeater, LBL-4235, Aug. 1975.

8. J.R. Alonso, C.A. Tobias, W.T. Chu, LBL-8947, March 1979. Also; following paper in these proceedings.

9. Ch. Leemann, J. Alonso, H. Grundel\%, E. Hoyer, G. Ka Inins, D. Rondeau, J. Staples, F. Voelker, IEEE Trans. Nucl. Sci. NS-24 (1977), p. 1052. 